\title{
Retorno ao esporte após reconstrução do LCA com ressecção ou preservação do remanescente*
}

\section{Return to Sports after ACL Reconstruction with Resection or Remnant-Preserving Technique}

\author{
Ruben Marcelo Maldonado Diaz ${ }^{1}$ () Fernando Cury Rezende ${ }^{1,2}$ Antonio Carlos Moscon ${ }^{10}$ \\ Carlos Eduardo da Silveira Franciozi ${ }^{2}{ }^{-0}$ Ana Luiza Cabrera Martimbianco ${ }^{1,3(0)}$ Aires Duarte Junior ${ }^{1,4}{ }^{\circ}$ \\ ${ }^{1}$ Grupo do Joelho da Ortocity, SP, Brasil \\ 2 Departamento de Ortopedia e Traumatologia, Universidade Federal \\ de São Paulo, São Paulo, SP, Brasil \\ 3 Programa de Pós-Graduação em Saúde e Meio Ambiente, \\ Universidade Metropolitana de Santos Santos, SP, Brasil \\ ${ }^{4}$ Departamento de Ortopedia e Traumatologia, Santa Casa de \\ Misericórdia de São Paulo, São Paulo, SP, Brasil \\ Endereço para correspondência Dra. Ana Luiza Cabrera Martimbianco,
Rua Brigadeiro Gavião Peixoto, 526, Lapa, São Paulo, SP, 05078-000,
Brasil (e-mail: analuizacabrera@hotmail.com). \\ Rev Bras Ortop 2020;55(4):432-437.
}

\section{Resumo \\ Palavras-chave \\ - lesões do ligamento cruzado anterior \\ - ruptura \\ - reconstrução do ligamento cruzado anterior \\ - retorno ao esporte \\ Objetivo Analisar os resultados da reconstrução do ligamento cruzado anterior (LCA) com preservação do remanescente, comparada à técnica convencional, no retorno do paciente ao nível de atividade física pré-lesão. \\ Métodos Estudo transversal retrospectivo, que avaliou indivíduos adultos submeti- dos à reconstrução anatômica do LCA no período de 2010 a 2014. Os desfechos analisados foram: nível de atividade física, taxa retorno ao esporte, relesão do LCA definida como lesão documentada que necessite de nova reconstrução ligamentar, e dor pela escala numérica de dor (EVN). \\ Resultados Foram incluídos 83 indivíduos com média de 31,8 anos de idade e segui- mento médio de 4,2 anos após a cirurgia, 34 submetidos à reconstrução do LCA com preservação do remanescente, e 49 à convencional. Não houve diferença estatisticamente significativa entre os grupos na frequência de atividade física pré-lesão e pós-operatória, na taxa de relesão do LCA reconstruído e na intensidade da dor no pós-operatório. Na análise intragrupos, houve uma queda estatisticamente significativa na frequência da prática de atividade física pós-operatória para ambos os grupos em comparação ao nível pré-lesão. 0 tipo de esporte mais praticado foi o futebol, onde $72 \%$ pacientes do grupo remanescente retornaram ao esporte comparado a $52,6 \%$ do grupo controle; porém, essa diferença não foi estatisticamente significante. \\ Conclusão Não foi possível observar diferenças entre os pacientes submetidos às técnicas cirúrgicas de reconstrução LCA com e sem a preservação do remanescente em relação ao retorno ao esporte, frequência de atividade física e intensidade da dor. Estudos futuros prospectivos são necessários.}

\footnotetext{
Trabalho desenvolvido no Grupo do joelho da Ortocity, São Paulo, SP, Brasil e Grupo do Joelho, Departamento de Ortopedia e Traumatologia, Universidade Federal de São Paulo, São Paulo UNIFESP/EPM, SP, Brasil.
}

recebido

24 de Janeiro de 2019

aceito

21 de Agosto de 2019
DOI https://doi.org/

$10.1055 / \mathrm{s}-0039-3402461$. ISSN $0102-3616$.
Copyright $\odot 2020$ by Sociedade Brasileira License terms de Ortopedia e Traumatologia. Published by Thieme Revinter Publicações Ltda, Rio de Janeiro, Brazil 
Abstract

\section{Keywords}

- anterior cruciate ligament

- rupture

- anterior cruciate ligament reconstruction

- return to sport
Objectives To analyze the results of anterior cruciate ligament $(\mathrm{ACL})$ reconstruction with remnant-preserving versus remnant-resecting technique, concerning the return to pre-lesion activity level.

Methods The present retrospective cohort study has assessed adults $>18$ years old who underwent ACL anatomical reconstruction between 2010 and 2014. The main outcomes assessed were: level of physical activity (4-point scale), sports participation rate, $A C L$ rerupture defined as documented lesion requiring revision surgery and the numeric pain scale rate (NPSR).

Results A total of 83 individuals were included in the study, with a mean age of 31.8 years old and follow-up mean time of 4.2 years after the surgery. A total of 34 patients underwent $A C L$ reconstruction with remnant-preserving technique, and 49 without remnant preservation. No statistically significant difference was found between groups in all outcomes assessed: level of physical activity before the lesion and after the surgery, ACL rerupture rates and postoperative pain level. Subgroup analysis has shown a statistically significant decrease in the activity level in both groups. The most practiced sport was football; $72 \%$ of patients in the remnant group have resumed football activity versus $52.6 \%$ of the control group.

Conclusion Based in these findings, the comparison between $\mathrm{ACL}$ reconstruction with remnant preserving technique and remnant resecting technique has shown no differences concerning the return to prelesion activity level.

\section{Introdução}

A reconstrução do ligamento cruzado anterior (LCA) do joelho visa restaurar a estabilidade articular do joelho, recuperar a capacidade funcional e esportiva no nível prévio à lesão, e ainda, prevenir a ocorrência de lesões meniscais e osteoartrite secundária. ${ }^{(1)}$ Entretanto, sabe-se que a restauração da função do joelho depende não apenas da técnica cirúrgica, mas de fatores anatômicos e biomecânicos envolvidos, e da interação entre os sistemas nervoso e musculoesquelético. ${ }^{1,2}$

Além de suas funções mecânicas, o LCA atua como órgão sensorial proprioceptivo devido à presença de mecanorreceptores em torno de suas fibras, os quais mantém a estabilidade articular do joelho por estimularem as contrações musculares coordenadas. ${ }^{2,3}$ Estudos histológicos revelaram a existência de mecanorreceptores residuais no tecido remanescente do ligamento lesionado, além do elevado potencial de cicatrização devido ao suporte vascular fornecido pela bainha sinovial intacta. Por esta razão, acredita-se que a preservação das fibras remanescentes do LCA possa auxiliar no processo de cicatrização biológica do enxerto e acelerar a cobertura sinovial do ligamento reconstruído. ${ }^{1,3-8}$

Tie et al. ${ }^{1}$ publicaram uma revisão sistemática em 2016 comparando a preservação do remanescente e a técnica convencional. Não houve diferença entre os grupos ao avaliar a estabilidade articular; entretanto, os autores demonstraram menor porcentagem de aumento do túnel tibial no grupo que manteve o remanescente. Apesar dos resultados clínicos satisfatórios observados em estudos recentes, ${ }^{1,6,9,10}$ ainda não está claro na literatura se a preservação ou ressecção deste tecido pode influenciar o risco de complicações funcionais no joelho, e influenciar o retorno do indivíduo para suas atividades esportivas. Sendo assim, o objetivo do presente estudo foi analisar os resultados da reconstrução do ligamento cruzado anterior (LCA) com preservação do remanescente comparada à técnica convencional, no retorno do paciente às atividades físicas, bem como na taxa de recidiva de lesão e intensidade da dor.

\section{Materiais e Métodos}

Trata-se de um estudo transversal retrospectivo, aprovado pelo Comitê de Ética e Pesquisa sob o número CEP/UNIFESP n: $1107 / 2016$.

Foram incluídos no presente estudo 83 pacientes adultos com idade $>18$ anos numa série consecutiva no período de 2010 a 2014, com o tempo médio de 4,2 anos de seguimento (mínimo de 2 e máximo de 7), 34 submetidos à reconstrução do ligamento cruzado anterior (LCA) com a preservação do remanescente, e 49 submetidos à reconstrução convencional. Foram excluídos os indivíduos com cirurgia prévia no joelho ou outras articulações, lesão condral de espessura total graus 3 ou 4 de acordo com a International Cartilage Repair Society (ICRS), meniscectomia maior que dois terços do menisco, presença de lesão concomitante em outros ligamentos do joelho (exceto ligamento colateral medial (LCM) grau 1 e/ou cirurgia ou lesão do membro inferior contralateral.

A técnica de reconstrução do LCA com preservação do remanescente do estudo era realizada para lesões parciais do LCA por meio da reconstrução seletiva com uma única banda (single bundle augmentation [SBA]), a qual consiste em preservar as inserções distal e proximal do coto do remanescente, posicionando o enxerto no sítio anatômico ("footprint") da banda que se encontrasse rompida (-Figura 1A e 1B). ${ }^{7,8} \mathrm{~A}$ 


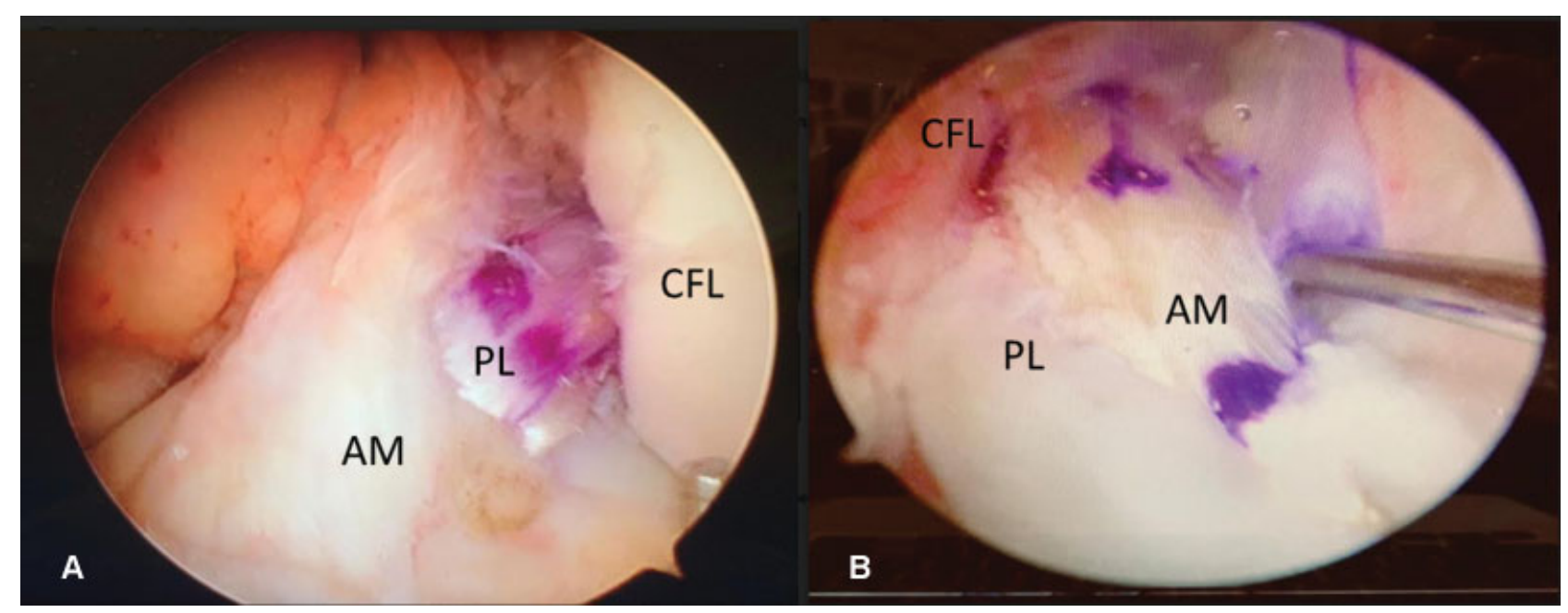

Fig. 1 (A) Reconstrução seletiva da banda posterolateral (PL) do ligamento cruzado anterior. (B) Reconstrução seletiva da banda anteromedial (AM) do ligamento cruzado anterior. CFL: côndilo femural lateral.

técnica de reconstrução do LCA sem preservação do remanescente era realizada por meio da limpeza minuciosa do intercôndilo com a retirada de todo remanescente do LCA.

Os pacientes selecionados tiveram seus dados coletados dos prontuários, descrição cirúrgica e dados adicionais e de seguimento foram coletados por contato pessoal ou telefônico, realizado por dois autores do presente estudo e com roteiro de entrevista e formulário padronizado para preenchimento dos dados. Os desfechos analisados foram: nível de atividade física (escala de 4 pontos: [1] atleta profissional, [2] atleta amador [>3/semana], [3] atleta recreativo [1 a $2 /$ semana], [4] sedentário), taxa de retorno ao esporte, relesão do LCA definida como lesão documentada que necessite de nova reconstrução ligamentar e dor pela escala numérica de dor (EVN; de 0 a 10 pontos).

Todas as análises estatísticas foram realizadas utilizando-se o programa STATA ${ }^{11}$ disponível comercialmente (STATA, version 13; StataCorp LP, College Station, TX, EUA). O nível alfa (erro tipo I) foi definido em 0,05 . A suposição de normalidade foi avaliada pela inspeção de histogramas e utilizando o teste de Shapiro-Wilk. Foi realizado o cálculo da média e desvio-padrão (DP) das variáveis contínuas (intervalo de confiança [IC] de 95\%), a frequência (porcentagem) para os dados dicotômicos e categóricos (IC de 95\%).
O teste qui-quadrado foi utilizado para comparação entre as variáveis da amostra, e o teste-t não pareado para comparar os grupos quanto às variáveis contínuas de distribuição normal. O teste não paramétrico de Mann-Whitney foi utilizado para comparar as variáveis contínuas de distribuição não normal. Foi considerado um IC de $95 \%$ para todos os testes.

\section{Resultados}

Ao todo, 71 pacientes $(85,5 \%)$ foram do gênero masculino, média de idade de $31,8( \pm 8,3)$ anos. 0 lado mais acometido foi o direito (65\%). Não houve diferença estatisticamente significativa nas características demográficas dos pacientes. Em relação ao tempo de retorno ao esporte, diferença no nível de atividade física no período pré-lesão e pós-operatório, taxa de relesão do LCA reconstruído e a EVN no pósoperatório, não foi observada diferença estatisticamente significativa entre os grupos ( - Tabela $\mathbf{1}$ ).

Não houve diferença estatisticamente significativa entre os grupos ao comparar a frequência de atividade física pré-lesão e pós-operatória (-Tabela 2). Contudo, foi observada diferença estatisticamente significativa na análise intragrupos da frequência de atividade física pré-lesão e pós-operatória para

Tabela 1 Distribuição da amostra de acordo com as características dos pacientes

\begin{tabular}{|l|l|l|l|}
\hline & $\begin{array}{l}\text { Grupo Convencional } \\
(\boldsymbol{n}=\mathbf{4 9})\end{array}$ & $\begin{array}{l}\text { Grupo Remanescente } \\
(\boldsymbol{n}=\mathbf{3 4 )}\end{array}$ & valor-p \\
\hline Idade (anos) (M $\pm \mathrm{DP})$ & $32,3( \pm 9,0)$ & $31,2( \pm 7,52)$ & 0,88 \\
\hline Lateralidade & $34 \mathrm{D} / 15 \mathrm{E}$ & $20 \mathrm{D} / 14 \mathrm{E}$ & 0,18 \\
\hline $\begin{array}{l}\text { Tempo de retorno às atividades físicas } \\
\text { (meses; M } \pm \mathrm{DP})\end{array}$ & $8,3( \pm 3,6)$ & $10( \pm 5,0)$ & 0,19 \\
\hline $\begin{array}{l}\text { Diferença no nível de atividade física } \\
\text { (Pré-lesão e PO; M } \pm \mathrm{DP})\end{array}$ & $-0,44( \pm 0,9)$ & $-0,46( \pm 0,9)$ & 0,74 \\
\hline Re-ruptura do neo LCA & 1 & 3 & 0,15 \\
\hline EVN PO (M DP) & $1,6( \pm 2,1)$ & $1,2( \pm 2,5)$ & 0,78 \\
\hline
\end{tabular}

Abreviações: D, direito; DP, desvio padrão; E, esquerdo; EVN, escala visual numérica; LCA, ligamento cruzado anterior; M, média; PO, pós-operatório. 
Tabela 2 Comparação entre a frequência de atividade física pré-lesão e pós operatória

\begin{tabular}{|l|l|l|l|}
\hline Frequência de atividade física & $\begin{array}{l}\text { Grupo Convencional } \\
(\boldsymbol{n}=\mathbf{4 9})\end{array}$ & $\begin{array}{l}\text { Grupo Remanescente } \\
(\boldsymbol{n}=\mathbf{3 4})\end{array}$ & valor-p \\
\hline Pré-lesão (N): & & & 0,24 \\
\hline (1) atleta profissional & 1 & 1 & \\
\hline (2) atleta amador (> 3/semana) & 24 & 12 & \\
\hline (3) atleta recreativo (1 a 2/semana) & 19 & 20 & 0,37 \\
\hline (4) sedentário & 5 & 1 & \\
\hline Pós-operatória (N): & & 1 & \\
\hline (1) atleta profissional & 0 & 6 & \\
\hline (2) atleta amador (> 3/semana) & 14 & 17 & \\
\hline (3) atleta recreativo (1 a 2/semana) & 19 & 10 & \\
\hline (4) sedentário & 16 & & \\
\hline
\end{tabular}

Abreviaçãp: N: número de pacientes.

Tabela 3 Análise intragrupo da frequência de atividade física pré- e pós-operatória

\begin{tabular}{|l|l|l|}
\hline $\begin{array}{l}\text { Frequência de } \\
\text { atividade física }\end{array}$ & $\begin{array}{l}\text { Grupo } \\
\text { Convencional } \\
(\mathbf{N}=\mathbf{4 9 )}\end{array}$ & $\begin{array}{l}\text { Grupo } \\
\text { Remanescente } \\
(\boldsymbol{N}=34)\end{array}$ \\
\hline Pré-lesão $(\mathrm{M} \pm \mathrm{DP})$ & $2,5( \pm 0,7)$ & $2,6( \pm 0,6)$ \\
\hline Pós-operatória $(\mathrm{M} \pm \mathrm{DP})$ & $3,0( \pm 0,7)$ & $3,0( \pm 0,7)$ \\
\hline valor-p & $<0,01^{*}$ & $<0,01^{*}$ \\
\hline
\end{tabular}

Abreviações: DP, desvio padrão; M, média.

ambos os grupos, demonstrando diminuição do nível de atividade física no pós-operatório (-Tabela 3).

O tipo de esporte mais praticado entre os pacientes foi o futebol. Dos 44 pacientes que praticavam este esporte antes da cirurgia, 25 (56,8\%) eram do grupo com preservação do remanescente e 19 (43,2\%) do grupo controle. Após a cirurgia com seguimento médio de 4,2 anos, 18 (72\%) pacientes do grupo remanescente retornaram ao futebol comparado a 10 $(52,6 \%)$ do grupo controle. Contudo, na comparação entre os grupos, não houve diferença estatisticamente significativa $(p=0,97)$.

\section{Discussão}

A técnica de reconstrução do ligamento cruzado anterior (LCA) com preservação do remanescente tem sido estudada nos últimos anos. Porém, os achados dos estudos continuam controversos e pouco se sabe sobre o índice de retorno às atividades físicas ao comparar esta técnica com a convencional. 0 presente estudo analisou de forma retrospectiva as técnicas de reconstrução do ligamento cruzado anterior (LCA), com e sem a preservação do remanescente, em relação ao retorno dos pacientes às suas atividades físicas prévias à lesão. Não foi observada diferença estatisticamente significativa entre os grupos em nenhum dos desfechos avaliados: frequência de atividade física pré-lesão e pós-operatória, taxa de retorno ao esporte, taxa de relesão do LCA recons- truído e intensidade da dor, no seguimento pós-operatório de 4,2 anos em média. Entretanto, ao considerar a análise intragrupos, ambos os grupos mostraram uma queda estatisticamente significativa, na frequência da prática de atividade física no período pós-operatório, quando comparados ao período pré-lesão.

O futebol foi a atividade atlética mais praticada pelos pacientes antes da lesão (53\%), fato esse que pode ser justificado por ser o esporte mais comum do nosso meio e pela sua grande exigência biomecânica para a articulação do joelho. Na análise de subgrupos avaliando apenas os indivíduos praticantes de futebol, não houve diferença na taxa de retorno ao esporte apesar do fundamento teórico da associação sinérgica entre reabilitação acelerada pós-reconstrução do LCA e preservação do remanescente já reportado em estudos prévios. ${ }^{7,8,12,13} \mathrm{Um}$ dos fatores que parecem otimizar a reabilitação nesses indivíduos com preservação do remanescente está relacionado à proteção mecânica ao enxerto promovida pelo coto do remanescente no pós-operatório precoce, não dependendo somente dos dispositivos de fixação na fase inicial de incorporação do enxerto. ${ }^{7,8,13}$ Além disso, a inervação proprioceptiva também potencializaria o retorno ao esporte, já que os indivíduos com fibras do remanescente intactas parecem apresentar maior senso de posição da articulação (joint position sense), o que se traduz em maior controle do membro na retomada da prática esportiva com pivoting. ${ }^{7,8,13}$ Entretanto, no presente estudo, não foi possível confirmar essa superioridade teórica do grupo com preservação do remanescente na avaliação do desfecho retorno ao esporte, o que torna necessária a realização de mais estudos clínicos com melhor refinamento metodológico para apurar tais achados.

A base teórica para a preservação do coto remanescente do LCA se justifica pela presença de mecanorreceptores, fibras proprioceptivas funcionais, vascularização subsinovial e intrafascicular neste tecido, identificáveis por meio de técnicas de histopatologia convencional e imunohistologia., ${ }^{2,14,15}$ Neste contexto, seria lógico pensar que o enxerto convencional, acrescentado pelo volume das fibras remanescentes com a presença de mecanorreceptores e vascularização subsinovial, 
forneceriam maiores vantagens em relação à técnica convencional. ${ }^{7,8}$ Apesar desta provável vantagem, os estudos clínicos de avaliação pós-operatória não têm demonstrado diferenças estatisticamente significativas entre as técnicas. ${ }^{9,16,17}$ Recentemente, uma revisão sistemática de ensaios clínicos randomizados ${ }^{18}$ mostrou que a preservação do tecido remanescente não é clinicamente superior à técnica convencional em termos de função física (avaliada pelo escore International Knee Documentation Committee [IKDC]), testes de estabilidade ligamentar (Lachman e Pivot-shift), amplitude de movimento do joelho e ocorrência de eventos adversos como a lesão tipo ciclope. Outra revisão sistemática publicada por Tie et al. ${ }^{1}$ demonstrou similaridade entre os grupos de reconstrução do LCA com e sem a preservação do remanescente, em relação à estabilidade anterior e à recuperação funcional do joelho. Houve menor alargamento do túnel tibial no grupo onde o tecido residual foi preservado.

Poucos estudos avaliaram efetivamente o retorno do paciente às atividades físicas prévias à lesão comparando-se à preservação ou ressecção do remanescente de LCA. Takazawa et al. ${ }^{19}$ realizaram um estudo caso-controle e, ao avaliar o nível de atividade física pelo escore de Tegner, não observaram diferença entre os grupos com e sem a preservação do remanescente após 1 ano de seguimento pós-operatório; mesmo assim, houve menor índice de relesão no grupo com preservação do tecido ligamentar residual e uma significativa melhora da estabilidade anterior. 0 presente estudo não constatou diferenças entre os grupos quanto ao retorno ao esporte ou relesão; no entanto, demonstrou uma importante diminuição na frequência da prática de atividade física no período pós-operatório, quando comparada ao período pré-lesão, independentemente da técnica utilizada. Sabe-se que o retorno ao esporte após a reconstrução do LCA é um fator preocupante, principalmente quando se compara o nível de atividade pré-lesão. A maioria dos estudos que analisa esses dados engloba pacientes com atividade esportiva de elite obtendo resultados superiores aos pacientes que praticam esporte em nível recreativo. Enquanto estudos envolvendo a elite esportiva reportam $80 \%$ de retorno ao esporte e $65 \%$ de retorno ao mesmo nível pré-lesão, estudos envolvendo em sua maioria pacientes com nível recreativo de atividade, como o presente trabalho, representam a maior parte dos pacientes submetidos à reconstrução do LCA e são necessários para o entendimento da evolução desses pacientes, normalmente menos favorável. ${ }^{20,21}$

O presente estudo apresentou como principais limitações o número reduzido da amostra, o desenho do estudo de caráter retrospectivo com a coleta dos dados realizada por contato telefônico e o tempo de seguimento insuficiente para avaliar desfechos de longo prazo. Apesar dessas restrições metodológicas, o presente estudo é um dos poucos a avaliar o retorno ao esporte após a reconstrução do LCA com e sem o tecido remanescente. Sendo assim, estudos prospectivos com maior rigor metodológico são necessários para comprovar os benefícios e malefícios desta técnica de reconstrução do LCA com preservação do tecido ligamentar residual, principalmente com ênfase no retorno do paciente às suas atividades físicas prévias.

\section{Conclusão}

Baseado nos achados deste estudo, não foi possível observar diferenças entre os pacientes submetidos às técnicas cirúrgicas de reconstrução do ligamento cruzado anterior (LCA) com e sem a preservação do remanescente em relação ao retorno ao esporte, frequência de atividade física e intensidade da dor. Não houve diferença entre os grupos em relação à taxa de recidiva da lesão. Estudos futuros prospectivos são necessários para esclarecer a real influência da preservação do tecido residual do LCA no retorno à atividade física pré-lesão.

\section{Conflito de Interesses}

Os autores declaram não haver conflito de interesses.

\section{Agradecimentos}

Agradecimentos ao Dr. Guilherme Conforto Gracitelli pelo auxílio na elaboração deste projeto de estudo e revisão do artigo, e à Dra. Carolina Pelegrini Barbosa Gracitelli pelo auxílio na análise estatística.

\section{Referências}

1 Tie K, Chen L, Hu D, Wang H. The difference in clinical outcome of single-bundle anterior cruciate ligament reconstructions with and without remnant preservation: A meta-analysis. Knee 2016;23(04):566-574

2 Dhillon MS, Bali K, Vasistha RK. Immunohistological evaluation of proprioceptive potential of the residual stump of injured anterior cruciate ligaments (ACL). Int Orthop 2010;34(05):737-741

3 Song GY, Zhang J, Li X, Chen XZ, Li Y, Feng H. Acute anterior cruciate ligament reconstruction with an augmented remnant repair: a comparative macroscopic and biomechanical study in an animal model. Arthroscopy 2014;30(03):344-351

4 Kazusa H, Nakamae A, Ochi M. Augmentation technique for anterior cruciate ligament injury. Clin Sports Med 2013;32(01): $127-140$

5 Muneta T, Koga H, Nakamura T, et al. A new behind-remnant approach for remnant-preserving double-bundle anterior cruciate ligament reconstruction compared with a standard approach. Knee Surg Sports Traumatol Arthrosc 2015;23(12):3743-3749

6 Gohil S, Annear PO, Breidahl W. Anterior cruciate ligament reconstruction using autologous double hamstrings: a comparison of standard versus minimal debridement techniques using MRI to assess revascularisation. A randomised prospective study with a one-year follow-up. J Bone Joint Surg Br 2007;89(09):1165-1171

7 da Silveira Franciozi CE, Ingham SJ, Gracitelli GC, Luzo MV, Fu FH, Abdalla RJ. Updates in biological therapies for knee injuries: anterior cruciate ligament. Curr Rev Musculoskelet Med 2014;7 (03):228-238

8 Luzo MV, Franciozi CE, Rezende FC, Gracitelli GC, Debieux P, Cohen M. Anterior cruciate ligament - updating article. Rev Bras Ortop 2016;51(04):385-395

$9 \mathrm{Hu}$ J, Qu J, Xu D, Zhang T, Zhou J, Lu H. Clinical outcomes of remnant preserving augmentation in anterior cruciate ligament reconstruction: a systematic review. Knee Surg Sports Traumatol Arthrosc 2014;22(09):1976-1985

10 Song GY, Zhang H, Zhang J, et al. The anterior cruciate ligament remnant: to leave it or not? Arthroscopy 2013;29(07):1253-1262

11 Stata, Corporation. Stata statistical software: release 7.0. 2001

12 Borbon CA, Mouzopoulos G, Siebold R. Why perform an ACL augmentation? Knee Surg Sports Traumatol Arthrosc 2012;20 (02):245-251 
13 Dejour D, Ntagiopoulos PG, Saggin PR, Panisset JC. The diagnostic value of clinical tests, magnetic resonance imaging, and instrumented laxity in the differentiation of complete versus partial anterior cruciate ligament tears. Arthroscopy 2013;29(03):491-499

14 Bali K, Dhillon MS, Vasistha RK, Kakkar N, Chana R, Prabhakar S. Efficacy of immunohistological methods in detecting functionally viable mechanoreceptors in the remnant stumps of injured anterior cruciate ligaments and its clinical importance. Knee Surg Sports Traumatol Arthrosc 2012;20(01):75-80

15 Sha L, Xie G, Zhao S, Zhao J. A morphologic and quantitative comparison of mechanoreceptors in the tibial remnants of the ruptured human anterior cruciate ligament. Medicine (Baltimore) 2017;96(05):e6081

16 Demirağ B, Ermutlu C, Aydemir F, Durak K. A comparison of clinical outcome of augmentation and standard reconstruction techniques for partial anterior cruciate ligament tears. Eklem Hastalik Cerrahisi 2012;23(03):140-144

17 Pujol N, Colombet P, Potel JF, et al. French Arthroscopy Society (SFA). Anterior cruciate ligament reconstruction in partial tear: selective anteromedial bundle reconstruction conserving the posterolateral remnant versus single-bundle anatomic ACL reconstruction: preliminary 1 -year results of a prospective randomized study. Orthop Traumatol Surg Res 2012;98(8, Suppl) S171-S177

18 Ma T, Zeng C, Pan J, Zhao C, Fang H, Cai D. Remnant preservation in anterior cruciate ligament reconstruction versus standard techniques: a meta-analysis of randomized controlled trials. J Sports Med Phys Fitness 2017;57(7-8):1014-1022

19 Takazawa Y, Ikeda H, Kawasaki T, et al. ACL Reconstruction Preserving the ACL Remnant Achieves Good Clinical Outcomes and Can Reduce Subsequent Graft Rupture. Orthop J Sports Med 2013;1(04):2325967113505076

20 Ardern CL, Taylor NF, Feller JA, Webster KE. Fifty-five per cent return to competitive sport following anterior cruciate ligament reconstruction surgery: an updated systematic review and metaanalysis including aspects of physical functioning and contextual factors. Br J Sports Med 2014;48(21):1543-1552

21 Waldén $M$, Hägglund $M$, Magnusson H, Ekstrand J. ACL injuries in men's professional football: a 15 -year prospective study on time trends and return-to-play rates reveals only $65 \%$ of players still play at the top level 3 years after ACL rupture. Br J Sports Med 2016;50(12):744-750 